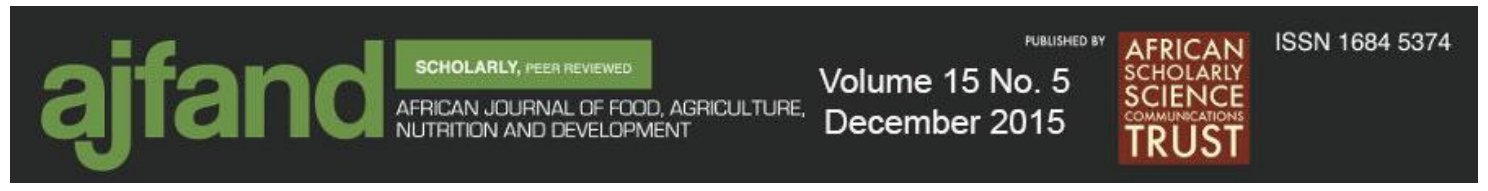

\title{
ENGAGING FATHERS AND GRANDMOTHERS TO IMPROVE MATERNAL AND CHILD DIETARY PRACTICES: PLANNING A COMMUNITY-BASED STUDY IN WESTERN KENYA
}

Thuita FM', Martin $\mathrm{SL}^{2}$, Ndegwa $\mathrm{K}^{3}$, Bingham $\mathrm{A}^{4 *}$ and Mukuria $\mathrm{AG}^{5}$

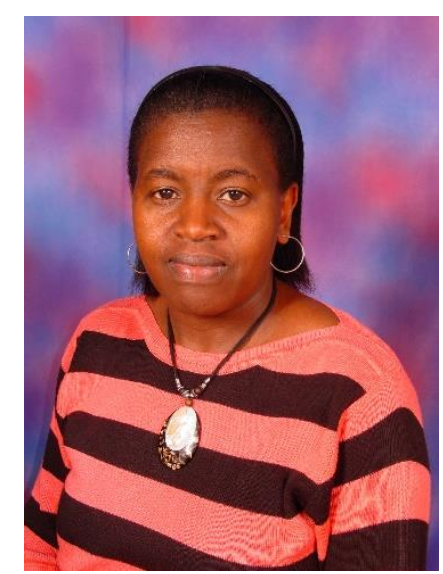

Faith Thuita

*Corresponding author's email: abingham@path.org

${ }^{1}$ School of Public Health, University of Nairobi, Nairobi, Kenya

${ }^{2}$ Division of Nutritional Sciences, Cornell University, Ithaca, New York, USA

${ }^{3}$ Independent consultant, PATH, Kenya

${ }^{4}$ PATH, Nairobi, Kenya

${ }^{5}$ Independent consultant, formerly with the Infant \& Young Child Nutrition (IYCN) Project 


\section{ABSTRACT}

Fathers and grandmothers are key family influencers who have an impact on maternal and child health. This paper describes the planning, design, and implementation of a four-phased evaluation study on the impact of engaging fathers or grandmothers in improving diets of mothers and feeding practices of infants and young children in a rural setting in western Kenya. The study used a quasi-experimental, non-equivalent comparison group design with pre- and post-test observations. It tested the hypothesis that families participating in activities to engage fathers or grandmothers have better knowledge and adopt better practices related to maternal nutrition and complementary feeding than families for which nutrition messages are targeted only to mothers. Information generated from previous formative research was used to design culturally relevant interventions for fathers and grandmothers. Interventions included separate but parallel peer education dialogue groups with fathers and grandmothers. They were held twice a month, and family bazaars and special fathers' days at local clinics were held once per month. The study team selected the dialogue-based group methodology, including promotion of social support actions, because it engages participants to actively discuss new information and experiences providing social support rather than passively receive information. Community health extension workers and Ministry of Health nutritionists provided supportive supervision and monitoring of the dialogue group activities. Father and grandmother interventions were implemented in two separate sub-locations of Kenya's former Western Province, and the program effects were compared to findings in a population with a similar socioeconomic background living in a similar sub-location in the same province. An examination of the study implementation methodology provides useful insights into practical issues that need to be addressed in programs seeking to engage key household influencers of maternal nutrition and infant and young child feeding behaviours. Results showed that the dialogue group methodology, formative research to inform intervention design, use of the existing Ministry of Health community health unit structure to provide critical support supervision, and actions that motivated peer mentors were key factors for successful implementation of the study intervention.

Key words: Maternal nutrition, infant feeding, complementary feeding, grandmother, father, design, formative research 


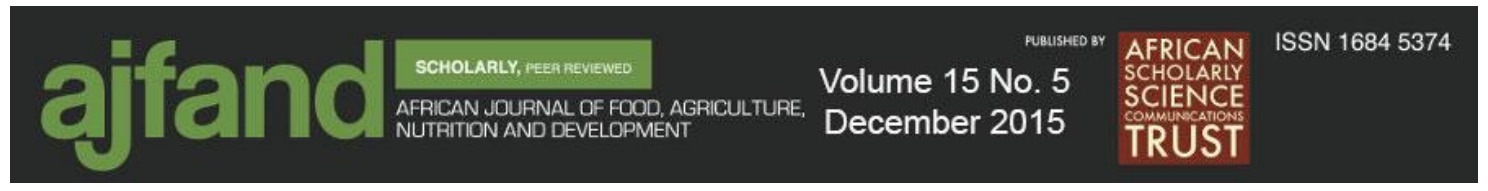

\section{INTRODUCTION}

In Kenya, poor breastfeeding and complementary feeding practices, coupled with high rates of childhood diseases, result in high rates of malnutrition and mortality during the first two years of life [1]. Results of the 2008/09 Kenya Demographic and Health Survey revealed persistently high levels of malnutrition, with stunting peaking at $46 \%$ among children in the second year of life [2]. While some gains have been made in the past five years, 2014 survey data show that still about $26 \%$ of Kenyan children are stunted, while 8 percent are severely stunted. Stunting is highest $(36 \%)$ in children age 18-23 months and lowest (10\%) in children age less than 6 months [3]. Although interventions promoting optimal breastfeeding have proved successful, feeding young children from six months of age has had less programmatic focus $[4,5]$.

Infant and young child feeding practices in Kenya were suboptimal in 2008. Only 63\% of children aged 6-23 months were fed the recommended minimum number of times for their age, and only $54 \%$ of young children were fed adequately diverse diets according to their age and breastfeeding status [2]. Although animal source foods and dark green leafy vegetables are a good source of iron, only $43 \%$ of children age 6-35 months consumed iron-rich foods in the previous 24 hours [2].

The national maternal, infant, and young child feeding strategy in Kenya notes a gap in locally tailored interventions targeting promotion of appropriate complementary feeding and maternal nutrition practices [6]. Community health and nutrition programs have historically targeted mothers with young children. However, a number of studies have shown that household members are important social influencers of mothers' infant feeding practices, including offering advice, providing food, and feeding children $[7,8]$. Some studies report that family members work to maintain cultural norms and practices, resisting adoption of new practices $[9,10]$. Other evidence has suggested that engaging grandmothers in group discussions, songs, and stories and engaging men in education activities can significantly improve breastfeeding and nutrition practices [1116]. Moreover, a series of assessments and surveys on infant and young child feeding in Kenya strongly suggest that success in improving these practices depends on effectively engaging key influencers, including fathers and grandmothers [17-21]. A study from western Kenya describing how infant feeding occurs within a family context criticised program approaches that treat women as individual actors, when women see themselves as enmeshed in social relationships that affect how they care for their children [6].

To explore the roles of fathers and grandmothers as potential positive influencers of infant and young child feeding practices in Kenya, the project team designed a multiphased, multi-method study. The study used a social ecological approach that considers not only the mother's knowledge and practices but also the social expectations and influences of her familial relationships, the availability of health facilities and services, community norms, and the health policy environment [22].

The study's aim was to assess whether increased support by key household influencers would improve mothers' complementary feeding practices. The study was carried out 
under the aegis of the US Agency for International Development (USAID)-funded Infant \& Young Child Nutrition (IYCN) Project. Its purpose was to evaluate the effectiveness of interventions that engaged fathers and grandmothers at the household and community levels to improve maternal dietary, infant and young child feeding practices. The study evaluated an approach to engage men and grandmothers in dialogue groups (DGs) as well as in related community activities. The team tested the hypothesis that families participating in activities to engage fathers or grandmothers in nutrition issues have better knowledge and adopt better practices related to maternal nutrition and complementary feeding than do families for which nutrition messages are targeted only to mothers.

This paper reports the methodology for the study, selected insights from the formative research [23] and its influence on intervention design. Insights from the process evaluation on participant experiences with the interventions are offered [unpublished data, 24]. Practical issues for programs seeking to engage grandmothers and fathers in community-based infant and young child nutrition support conclude the paper.

\section{MATERIALS AND METHODS}

The study was implemented between June 2010 and July 2012. The methodological approach used to design, implement, and evaluate the intervention consisted of four phases (Figure 1):

1. Qualitative formative research to understand knowledge, practices, social relations and responsibilities within families to inform the intervention design

2. Quantitative baseline survey to collect information about nutrition knowledge and practices among mothers, fathers, and grandmothers

3. Community-based interventions to engage fathers and grandmothers in improving nutrition with a process evaluation to document participants' experiences

4. Quantitative endline survey to evaluate changes in knowledge, practices, dietary intake, feeding practices, and social support

\begin{tabular}{|c|c|c|c|c|c|c|c|c|c|}
\hline $\begin{array}{c}\text { June } \\
2010\end{array}$ & $\begin{array}{c}\text { November } \\
2011\end{array}$ & $\begin{array}{c}\text { December } \\
2011\end{array}$ & $\begin{array}{c}\text { January } \\
2012\end{array}$ & $\begin{array}{c}\text { February } \\
2012\end{array}$ & $\begin{array}{c}\text { March } \\
2012\end{array}$ & $\begin{array}{c}\text { April } \\
2012\end{array}$ & $\begin{array}{c}\text { May } \\
2012\end{array}$ & $\begin{array}{c}\text { June } \\
2012\end{array}$ & $\begin{array}{c}\text { July } \\
2012\end{array}$ \\
\hline $\begin{array}{c}\text { Formative } \\
\text { Research }\end{array}$ & \multicolumn{2}{|c|}{$\begin{array}{c}\text { Baseline } \\
\text { survey }\end{array}$} & \multicolumn{10}{|c|}{$\begin{array}{c}\text { Process } \\
\text { evaluation }\end{array}$} & $\begin{array}{c}\text { Endline } \\
\text { survey }\end{array}$ \\
\hline & & & \multicolumn{3}{|c|}{ Father and grandmother dialogue group interventions } \\
\end{tabular}

\section{Figure 1: Study phases and timelines}

\section{Study design}

The study team used a quasi-experimental, non-equivalent comparison group design with pre- and post-test observations to evaluate the impact of engaging fathers or paternal grandmothers (mothers-in-law) on maternal dietary, infant and young child feeding practices of mothers in Vihiga County in western Kenya (Figure 2). Interventions were implemented in two sub-locations, and program effects were 


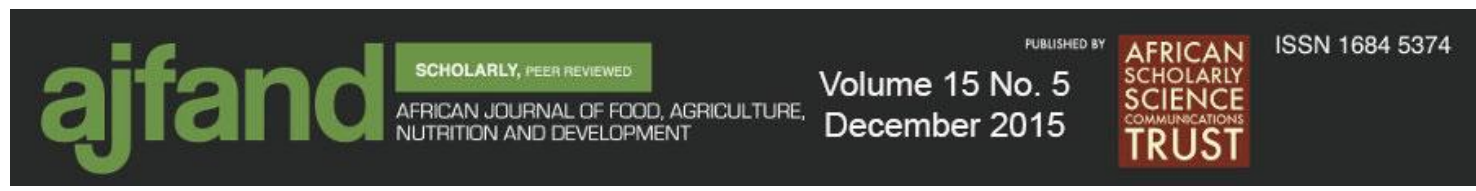

compared against findings in a population in a similar sub-location in the same county. Both intervention groups had pre- and post-intervention tests with the same participants. The comparison group was tested twice without any intervention activities.

\begin{tabular}{|l|l|l|l|}
\hline & Pre-test & Intervention & Post-test \\
\hline Arm 1: Intervention group & $\mathrm{O}_{1}$ & Father engagement & $\mathrm{O}_{2}$ \\
\hline Arm 2: Intervention group & $\mathrm{O}_{1}$ & $\begin{array}{l}\text { Grandmother } \\
\text { engagement }\end{array}$ & $\mathrm{O}_{2}$ \\
\hline Arm 3: Comparison group & $\mathrm{O}_{1}$ & No intervention & $\mathrm{O}_{2}$ \\
\hline
\end{tabular}

Figure 2: Study design

The study focused on the impact of engaging grandmothers and fathers as key household influencers of infant and child feeding practices. Through this study design we evaluated whether engagement through Dialogue Groups and community activities would translate to increased social support to mothers and subsequently improved feeding practices of infant and young children. To determine the impact of the intervention on fathers and grandmothers, the study team evaluated their knowledge of optimal maternal, infant and young child nutrition (MIYCN) and the social support they provided to mothers in all three sub-locations. Measures of social support included selected physical and material support that is generally provided during the early infant and young child period, such as help with household chores, provision of child care and provision of foodstuffs. Mothers in the targeted communities were not engaged in DG activities but did receive initial counseling and could participate in any community activity. They were surveyed at baseline and endline to assess knowledge and practices (Figure 3). 

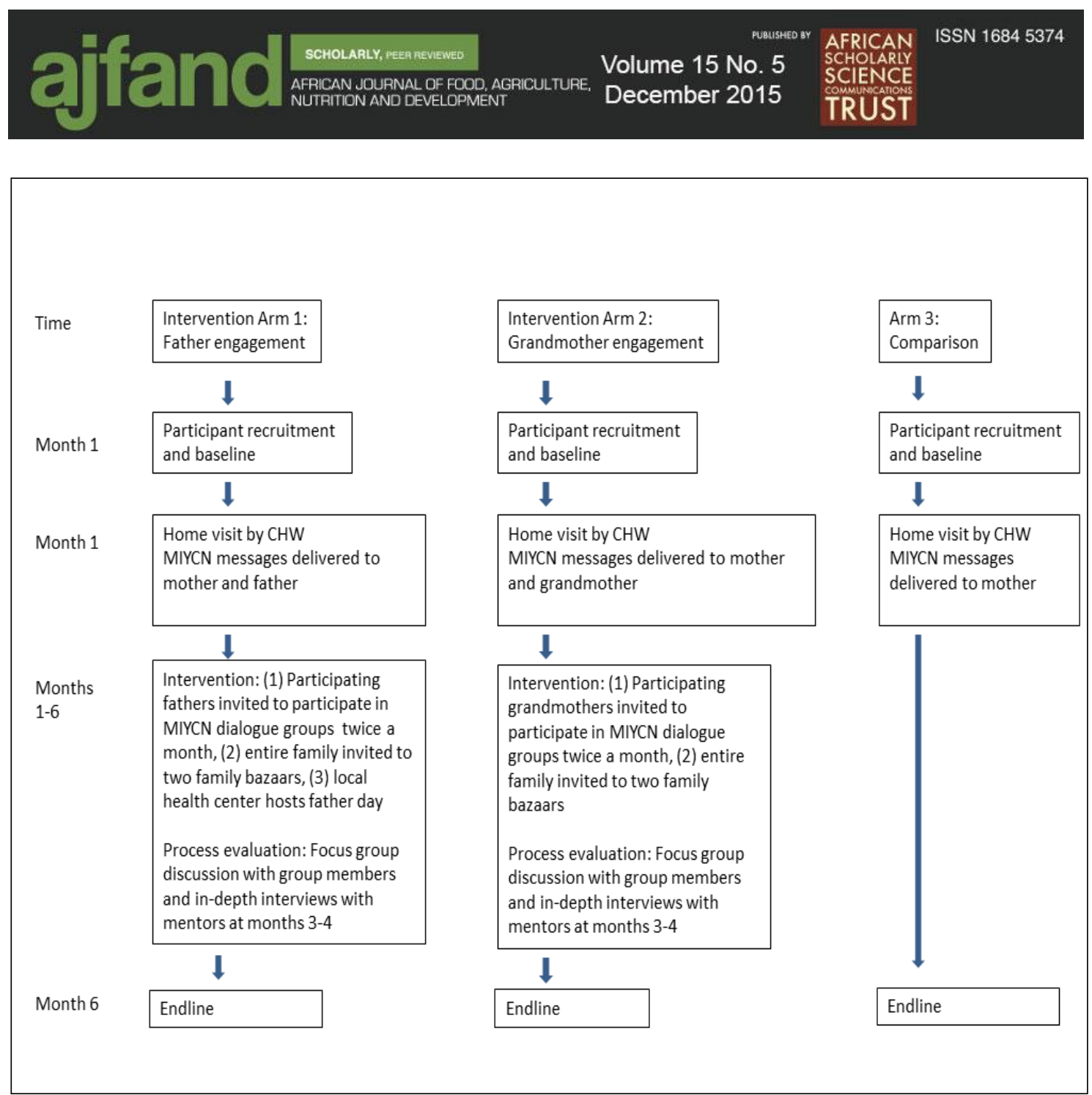

Figure 3: Intervention flow diagram

\section{Study sites}

The study was conducted in three sub-locations in three districts in Vihiga Countyone intervention area for fathers, one for grandmothers, and a comparison area. Study areas were selected based on consultations with personnel from the Ministry of Health $(\mathrm{MOH})$ and staff from the USAID-funded AIDS, Population and Health Integrated Assistance Plus (APHIAplus) Western Kenya Project. Selection criteria included the presence of a community health unit (CHU) and presence of APHIAplus Western Kenya community-level activities. Subsequent baseline survey findings showed that the three areas were similar culturally, socially, economically, and vis-à-vis livelihoods of the inhabitants. The comparison area, Mambai sub-location in Emuhaya District, was approximately $35 \mathrm{~km}$ from the intervention areas and had a low likelihood of being affected by spillover from study sites. The selected intervention area for fathers was Kitagwa sub-location in Hamisi District and for grandmothers was Viguru sub-location in Vihiga District.

\section{Study protocols and tools}

The research protocol and data collection tools for the formative assessment, process evaluation, and baseline and endline studies are discussed below. 


\section{Formative assessment}

The study team conducted a literature review and a qualitative formative assessment to understand maternal, infant, and young child nutrition knowledge and practices among mothers, fathers, and grandmothers and to inform intervention design and implementation [21]. The roles and responsibilities of fathers and grandmothers with regard to dietary and feeding practices, as well complementary feeding practices for children less than two years of age, maternal dietary practices, and the cultural beliefs and influencers of these behaviours were determined. The assessment used a participatory, in-depth approach to gather information from community members and selected key informants. Data were collected through both focus group discussions (FGDs) and key informant interviews (KIIs).

Four FGDs were held with fathers and four with grandmothers of children less than two years of age. To document findings, taped discussions were transcribed, translated, and used together with detailed notes. Fourteen KIIs were held with $\mathrm{MOH}$ officers, community and religious leaders, women's group leaders, and community health extension workers (CHEWs) and interviewers taking extensive notes. Discussions and interviews explored feasible and culturally relevant ways of engaging men and grandmothers in nutrition interventions. Findings from the literature review and formative research informed the development of the study protocol, instruments, and subsequent interventions.

\section{Process evaluation}

Mid-way through the study, the team conducted a process evaluation at the intervention sites. The evaluation included in-depth interviews with DG mentors (seven father mentors and ten grandmother mentors) and FGDs with father and grandmother DG members (8 and 10 FGDs, respectively).

\section{Baseline and endline evaluations}

The quantitative baseline and endline evaluations used separate questionnaires for mothers, fathers, and grandmothers. The questionnaires were translated into Swahili and back-translated by a different team to ensure that the original meaning was retained.

Baseline survey: Interviewers administered a baseline survey in November and December 2011 to assess knowledge and practices related to maternal nutrition and infant and young child feeding. The survey also evaluated social support provided to mothers by fathers and grandmothers about the care and feeding of young children.

The research assistants who conducted the survey were trained for three days in interviewing techniques, field data collection procedures, and use of the questionnaires. All had a secondary education, were from western Kenya, were fluent in the local language and Swahili, and had prior experience in field data collection. After the training, pilot testing was done in a sub-location that was not sampled for the study. 
Endline evaluation: After six months of intervention, the study team conducted follow-up surveys of grandmothers, fathers, and mothers in the same houses as the baseline. The evaluation measured: (1) maternal, infant, and young child nutrition knowledge and practices; (2) maternal and child dietary intake based on 24-hour and seven-day recalls; and (3) social support provided to mothers by fathers and grandmothers. Interviews lasted 30 to 45 minutes. Fathers and grandmothers were surveyed to determine any changes in their knowledge and support that could have contributed to changes in mothers' behaviours and practices.

Ethical considerations: Study protocols and tools were approved by PATH's Research Ethics Committee and the Kenyatta National Hospital/University of Nairobi ethics review committee. The consent form was translated into Swahili and administered by trained research assistants. Once a family was determined to be eligible for participation, the mother received the consent form to review and was given an opportunity to ask questions. Fathers and grandmothers of infants whose mothers had agreed to participate also gave their consent to be included in the study. Verbal consent was obtained before recording any FGD or in-depth interview. Audio recordings and transcripts from FGDs and individual interviews did not include identifying information.

\section{Intervention approach, materials, and training Training materials and supporting resources}

The study team developed a training curriculum, discussion guides, and an intervention delivery protocol that were used to train community health workers (CHWs) and DG mentors. Key messages, learning objectives, and content (Table 2) were generated based on findings from the literature review and formative assessment, project IYCN materials, $\mathrm{MOH}$ counselling materials, and WHO nutrition guidelines [25-28]. Key among these were the roles of grandmothers and men in maternal diets, young child nutrition, and health promotion; gender roles and division of labour and child care in the home; and effective communication in the family.

Facilitation and delivery methods employed widely recognised adult learning methodologies, including participatory learning activities that encourage reflection and are relevant to participant experiences. Consistent with the dialogue approach, discussions were carefully designed to stimulate critical inquiry and encourage reflection.

\section{Training of trainers}

The study team trained three $\mathrm{MOH}$ nutrition officers for three days on participatory training methods and group facilitation, optimal infant and young child feeding practices, maternal nutrition, and the use of counselling materials. These individuals subsequently trained $25 \mathrm{CHWs}$ and 2 CHEWs. Three of the CHWs were also CHU chairmen from the three study sites.

\section{Participant selection}

CHWs conducted a rapid census of households with infants in the intervention and comparison areas. Eligible households had mothers at least 18 years old and infants 6-9 


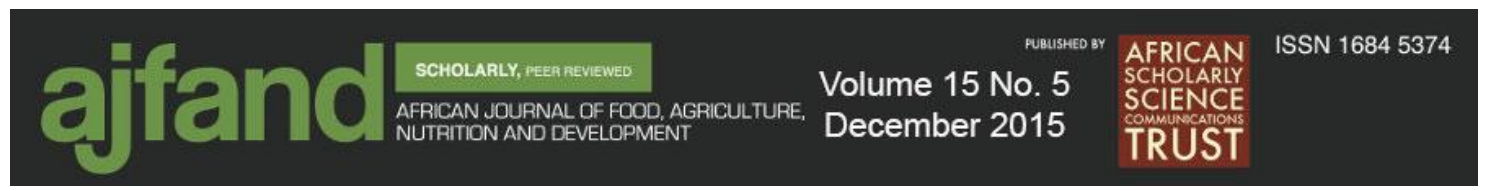

months of age at enrolment. All eligible households were invited to participate, and participation was voluntary. Individuals who were seriously ill and pregnant women were excluded. Table 1 shows the number of participants in the intervention and comparison areas.

\section{Interventions and monitoring}

Initial home visit: Trained CHWs conducted home visits with study participants in both the intervention and comparison groups and shared oral and written complementary feeding and maternal nutrition messages. In the intervention areas, fathers or grandmothers were invited to participate in this visit. In the comparison area, these messages were transmitted only to mothers. In all three sub-locations, CHWs left a brochure with key messages for each participant at the end of the visit.

Father and grandmother DGs: DGs gave fathers and grandmothers an opportunity to acquire knowledge, communication skills, and nutrition-related behavioural skills to enable them to support improvements in maternal diets and infant and young child feeding practices in their homes. Each DG had 8 to 12 members. Eight father DGs and ten grandmother DGs were formed in Kitagwa and Viguru sub-locations, respectively. In each DG, members selected a mentor to facilitate sessions. Group members set criteria for selection of group mentors, who were intended to be respected by the community, kind hearted and sociable, able to read and write, a good role model, and good communicator. Mentors received five days of training on health, nutrition, and social support topics; use of the training manual, discussion guides, and materials; and group facilitation techniques. Three $\mathrm{MOH}$ nutrition officers who had previously been trained by the IYCN study team conducted the training.

Dialogue group meetings were held twice a month at times and venues agreed upon by group members. Grandmother DGs generally met in members' homes. Father DGs typically met in churches, schools, or homes, often on Sunday afternoons (to avoid conflicts with work commitments). Tea allowance was provided to all groups during meetings, however, fathers preferred money (100 ksh/ 1 USD).

Community Health Workers (CHWs) supported peer educators/mentors during each meeting and assisted in answering questions. The mentors used a standard monitoring tool to record attendance, questions, or issues that were a challenge, as well as topics discussed.

\section{Community mobilisation and other activities}

Family bazaars: Two well-attended, half-day community bazaars in each area convened DG members, CHWs, community members, religious and local leaders, APHIAPlus Western Kenya staff, representatives of development partners, $\mathrm{MOH}$ officers, and Division of Nutrition (national and provincial) representatives. Information learned during DG meetings was expressed in the form of song, dance, skits, recitals, and testimonies from fathers, mothers, grandmothers, CHWs and even children. Mothers spoke of improved relationships, especially with their mothers-inlaw. Wives of participating men praised the increased support they received from their husbands, especially the improved provision of nutritious foods and help around the 
house. Participating fathers urged other men to take more responsibility for the health of their wives and children-especially around providing fruits and animal-based proteins to complement starch-based staples, ensuring infants and young children are breastfed for an appropriate duration, and assisting mothers with household chores. Speeches by government officials and representatives of community organisations called for community support of these efforts. Finally, a food demonstration and information tent showcased locally available foodstuffs and methods of food preparation to provide nutritious foods for children 6-23 months and breastfeeding mothers.

"Fathers' Days" at local clinics: To increase male understanding of, and comfort with maternal and child health services, each local clinic hosted five special "Fathers' Days." On these days, DG fathers accompanied their wives and children to the clinic and received information and messages on child health and nutrition. They also participated in growth monitoring sessions and were given the opportunity to ask questions to health care providers. The CHWs and CHEWs welcomed the fathers and answered their questions.

\section{Supervision and monitoring}

The CHWs and CHEWs provided close supervision and monitoring of the father and grandmother DGs. The CHWs attended all group meetings and helped answer questions as needed. They monitored the conduct of DG members and the depth and accuracy of the information discussed.

Each intervention area had two CHEWs, who oversaw the CHWs and DGs. Group meetings were regularly attended by CHEWs and occasionally by the District Nutritionist, who used a checklist for documenting general conduct of the meetings, levels of participation, and the quality of facilitation. This process supported the mentors by giving them feedback on their general performance and by providing technical support in addressing difficult questions. Monthly review meetings were held with group mentors, CHWs, CHEWs, and the District Nutritionist. The study coordinator attended about $70 \%$ of these meetings. Operational issues and challenges were discussed and addressed during the review meetings. Monitoring tools included attendance registers and DG reporting forms. Mentors used the reporting forms to record the topics, key lessons learned, and questions asked during the meeting.

\section{Data integrity}

The quality of the collected data was ensured through careful recruitment and training of research assistants and rigorous supervision of field data collection by trained supervisors. Thorough preparation of study teams included detailed review of data collection tools, discussion of field data collection techniques and procedures, and roleplaying in administration of questionnaires.

During the baseline and endline surveys, field supervisors closely monitored the work of the research assistants. Daily debriefing sessions addressed any challenges. The study team leader provided feedback for continual improvement of the process and quality assurance. Supervisors reviewed completed questionnaires on a daily basis to 


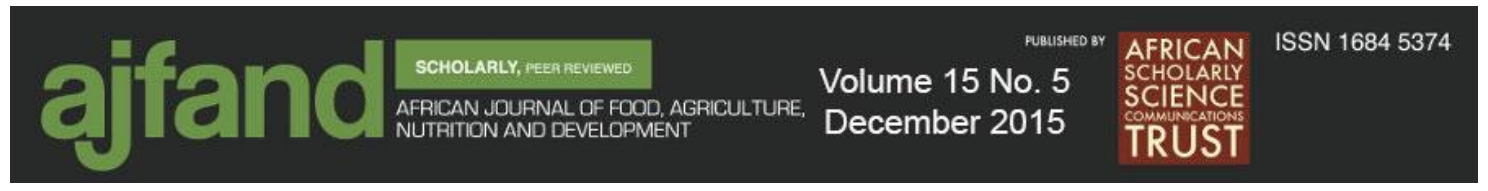

assess completeness. Once verified as complete, questionnaires were given to the study coordinator for safekeeping at the PATH office in Nairobi. Data collection took no more than four days in each area.

\section{RESULTS}

This section presents insights drawn from the formative assessment that influenced the intervention design [23]. Data from the process evaluation provide insights on practical issues for the delivery of social support interventions [unpublished data, 24]. The sections that follow include a reflection on the public health implications of these data and recommendations.

\section{Formative research findings}

Key informants provided valuable information about culturally acceptable ways of engaging men and grandmothers to improve maternal diets and feeding practices of young children. For instance, the study team found that men typically provide starchy foods, such as maize and maize meal, and animal protein while mothers and grandmothers often give fruits and vegetables to families. Findings of the formative assessment also showed that besides caring for young children, grandmothers strongly influence what is cooked (recipes used) and fed to young children in families. Discussants in all groups noted that senior women are key influencers in making household decisions about maternal nutrition, pregnancy, delivery, newborn care, and complementary feeding. This information informed design of the study tools and interventions for men and grandmothers.

Equally, while fathers are not directly involved in infant and young child feeding, they play a critical supportive role, providing food for the child and mother, and key financial and logistical resources for health care. Findings also showed that fathers were receptive to well-informed people as trainers and leaders, as well as engagement in line with their cultural roles as providers, such as asking them to provide animalsource foods to mothers and children. Fathers preferred to be educated through bazaars and seminars. Men also preferred meeting in social venues rather than in homes. (Note: Following the formative research, when the DG concept was introduced to men, they embraced it but, preferred fathers-only groups with fathers-as-leaders.) They also noted that employment opportunities were central to their ability to provide animal-sourced foods to their families. Finally, both grandmothers and men expressed challenges in their interpersonal communication in the family. The training for group mentors in both grandmother and father interventions was tailored to address these concerns.

\section{Process evaluation findings}

Father and grandmother DG mentors described factors that contributed to the successful implementation of their groups during in-depth interviews conducted as part of the process evaluation. Mentors reported receiving sufficient training to facilitate their groups and appreciating the discussion guides provided. Mentors valued CHW support and participation during bi-weekly DG meetings, and the monthly mentor meetings appeared to be especially helpful for clarifying questions, sharing experiences, group problem solving, and sustaining motivation [24]. 
Mentors also observed changes in their group members' attitudes and behaviors, which they attributed to participation in the DGs. They also shared that their group members experienced improved relationships with spouses or daughters-in-law and provided increased support for recommended infant feeding practices [unpublished data]. The mentors and group members were widely respected in their communities, and, both reported others in the community coming to them for health and nutrition advice and wanting to join their groups [24].

In focus group discussions, father DG members reported providing nutritious foods for their families, helping their wives with chores and child care, accompanying their wives and bringing their children to the health center, and enjoying learning about infant feeding. Grandmother group members reported improved hygiene and child feeding practices, and appreciated being involved in the DGs and recognized for their role as infant feeding advisors within their families and communities. Cooking demonstrations with locally available foods were cited as important for understanding what to feed young children. In addition, group members appreciated being able to choose their DG mentors, most participants were satisfied with their mentors' performance.

Although participants stated they were very satisfied with their participation in the DGs, they offered suggestions for improvement. Some fathers and grandmothers stated they appreciated the tea allowance, but they needed additional resources and support. Several groups started "merry-go-round" savings plans as part of their groups, and others discussed the need for additional income generating activities or agriculture interventions to better enable them to improve infant feeding practices within their families. In addition, several participants and mentors continued to express confusion about HIV and infant feeding recommendations and requested additional training on this content [24].

\section{DISCUSSION}

Drawing from formative and process evaluation findings, we discuss key factors that led to the successful implementation of a social support intervention to improve infant and young child feeding practices. We discuss their wider relevance for programs to target fathers and grandmothers to support maternal and child nutrition practices.

Although research has shown that peer support interventions are effective in promoting breastfeeding [29, 30], evidence on the importance of social support interventions on complementary feeding practices is more limited [31]. Both the formative research and process evaluation findings confirmed that grandmothers play a leading role in making household decisions about maternal nutrition, pregnancy, delivery, newborn care, and complementary feeding. Fathers also play a key supportive role, providing financial and logistical resources for health care, and resources needed to ensure greater dietary diversity. 
A locally tailored intervention informed by a formative assessment does improve the acceptability of an intervention among beneficiaries. Although formative research is an important step in the design of effective community-based health and nutrition interventions, few studies report how formative research has been used to design programs and interventions and contributes to their successful implementation [32-35]. The formative research findings provided the basis for interventions targeting fathers and grandmothers, who were identified as key influencers of maternal nutrition and infant and young child feeding. The formative assessment also helped identify cultural norms for the roles and responsibilities of mothers, fathers, and grandmothers; determine typical foods consumed in the community and the dietary practices of women, infants and young children; reveal health care practices; and identify caregivers' sources of nutritional advice. In addition, both grandmothers and men expressed challenges. The training for DG mentors was tailored to address these issues.

The selection of a dialogue-based group methodology led to the successful engagement of fathers and grandmothers. PATH has successfully used this approach in western Kenya, India, and Nepal [36]. Participants learn to exchange information among their peers rather than 'transmit' information, a powerful process that can diffuse new information quickly through existing community networks, in this case, a mother's key familial network [35]. This interpersonal communication approach engages participants to actively discuss new information, rather than passively receive information. It promotes greater openness and discussion among families, couples, and peer networks, resulting in positive health-related outcomes [36, 38, 39].

The process evaluation revealed important insights regarding effective means for engaging men in this intervention. For example, although the formative research indicated that men prefer to receive information about health from trained professionals and not peers, process evaluation data suggest the DG process was acceptable to men. One primary reason was in part due to the in-depth training of the mentors and the support and regular attendance of the CHWs and CHEWs. Expanding nutrition interventions to include men is important [40], yet the most effective strategies to engage men in complementary feeding interventions have not been identified [15]. Our findings are an important contribution.

Highly motivated peer educators contributed significantly to DG activities. Their role in the successful implementation, sustainability, and scalability of community-based, peer-led nutrition interventions was the focus of an in-depth analysis [25].

Community health workers were also pivotal to the success of the DGs through ongoing technical support provided to mentors during DG meetings and monthly mentor meetings. Also, others have shown that to maintain a government-sponsored social support intervention delivered by volunteers, it is important to ensure that group mentors receive ongoing support and encouragement [41].

Use of existing infrastructure should be a central design feature and will likely increase the sustainability of community-based intervention [38]. In this study, use of the $\mathrm{MOH}$ CHUs - Kenya's first level of health care service delivery - was a major factor in the 


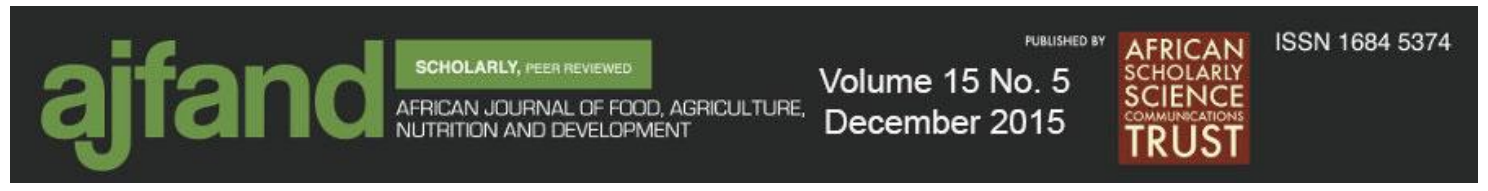

intervention's success. The CHU facilitated the research team's work at all study sites, organised community mobilisation events such as the family bazaars, provided meeting venues, and identified CHWs to be trained in consultation with the CHEWs.

Finally, using a family-centered approach should be a key feature of approaches to improve maternal and young child health and nutrition. Although this study engaged fathers and grandmothers separately, we would expect that engaging mothers, fathers, and grandmothers together would lead to even better results.

\section{CONCLUSION}

This paper has outlined the steps involved in planning, implementing, monitoring, and evaluating a community-based infant feeding social support program. Formative research findings informed design of the intervention to engage fathers and grandmothers in MIYCN. The process evaluation showed that, motivation levels of father and grandmother peer educators were influenced in part by key design factors. Reflections on our experience reveal key practical issues, that need to be addressed by programs seeking to engage household influencers of MIYCN. These include using existing community level structures, a dialogue-based group approach, allowing groups to select their leaders, establishing a system of support and active follow up through linkages with agency sponsors such as Kenya's Ministry of Health, and designing interventions informed and guided by gender roles. Results on maternal and child nutrition impact were extremely positive and publication is forthcoming.

The Kenya Demographic Health Survey 2014 notes the following [3]:

"In Kenya, about two in ten (21 percent) children ages 6-23 months consume an acceptable diet. Children age 12-17 months are slightly more likely than children in other age groups to consume an acceptable diet (24 percent)."

Scale up of effective, locally tailored and acceptable MIYCN interventions remains an essential priority in Kenya.

\section{ACKNOWLEDGEMENTS}

This study was funded through the IYCN Cooperative Agreement No. GPO-A-00-0600008-00 and the USAID Cooperative Agreement No. AID-623-A-11-00002. We are grateful for the technical assistance provided by the Kenyan Ministry of Health and the USAID-funded APHIAPlus Western Kenya Project. Strong program support was provided by PATH staff - especially Denise Lionetti, Rikka Trangsrud, and Janet Shauri. We acknowledge the editorial support provided by Marge Murray, John Ballenot, and Debjeet Sen. We also wish to thank the research team, including the research assistants, CHWs, and CHEWs for their hard work and dedication. Finally, we thank the communities in Viguru, Kitagwa, and Mambai sub-locations of Kenya's Western Province for participating in this study and enabling the exercise to be carried out fruitfully. 


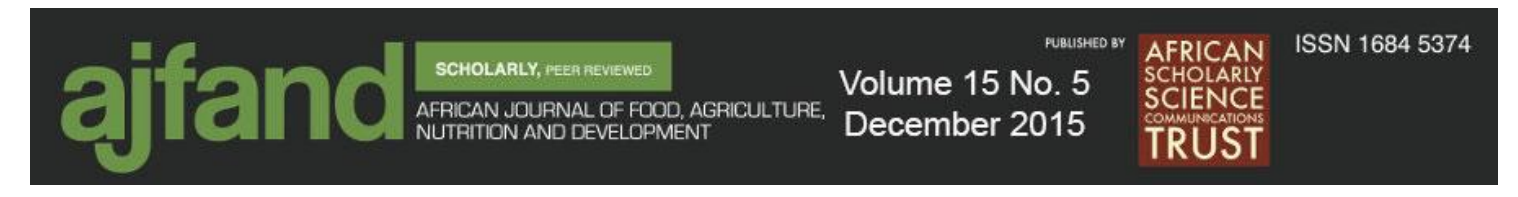

Table 1: Sample size



(ㄷ) $(1) \&(9)$ 


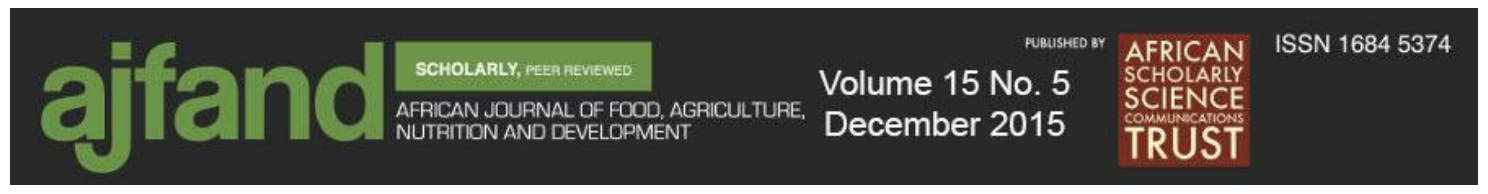

Table 2: Topics for dialogue group members training

\begin{tabular}{|c|c|}
\hline Father mentor training topics & Grandmother mentor training topics \\
\hline 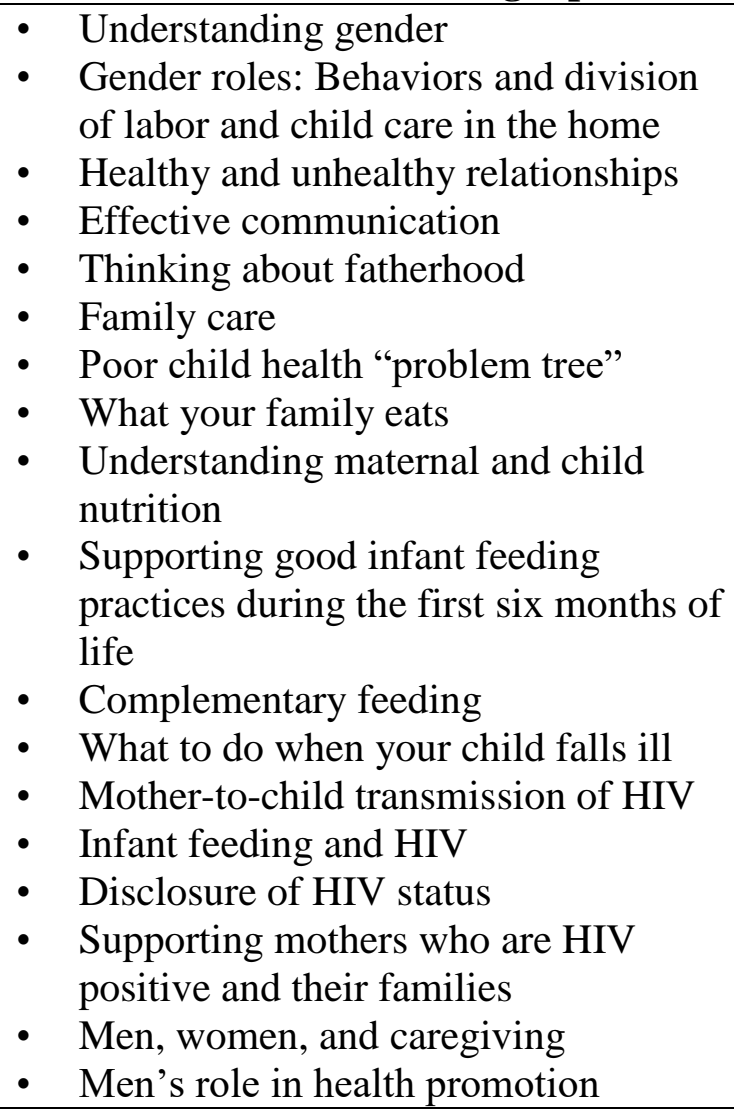 & 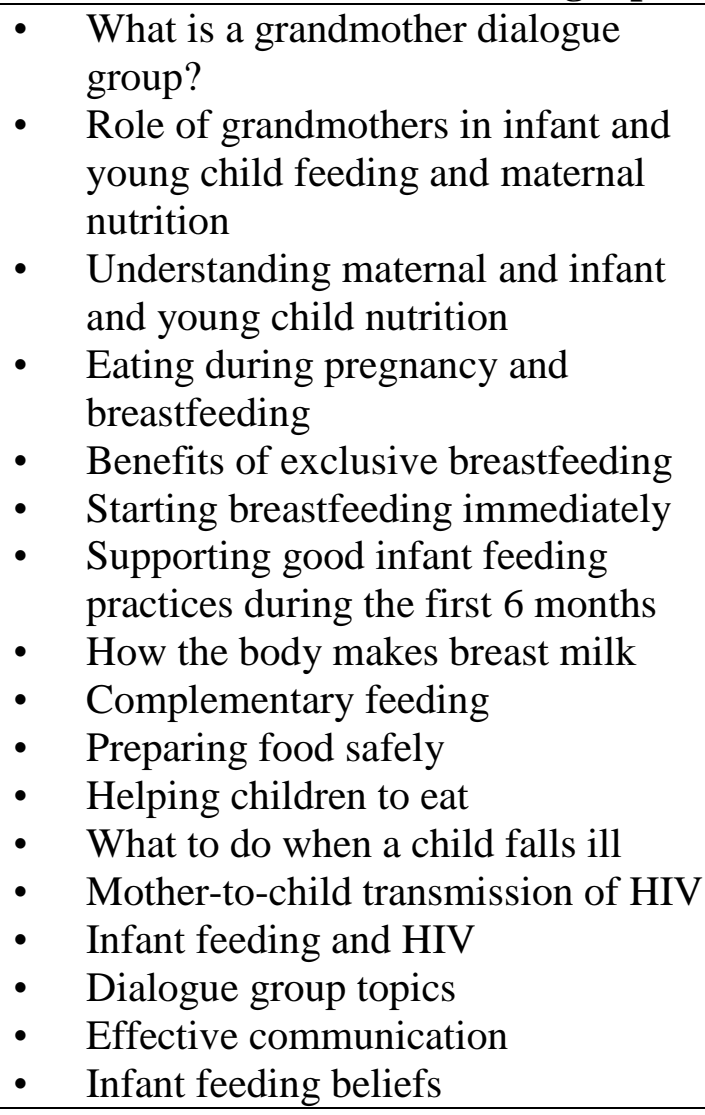 \\
\hline
\end{tabular}






\section{REFERENCES}

1. United Nations Children's Fund (UNICEF), State of the world's children, 2008. Eastern and Southern Africa Regional Office, 2007 www.unicef.org/sowc08/docs/sowc08.pdf. Accessed August 11, 2014.

2. Kenya National Bureau of Statistics (KNBS) and ICF Macro Kenya Demographic and Health Survey 2008-09. Calverton, Maryland: KNBS and ICF Macro, 2010.

3. Kenya National Bureau of Statistics (KNBS) Kenya Demographic and Health Survey 2014: Key Indicators. Nairobi, Kenya: KNBS and ICF Macro, 2015 http://dhsprogram.com/what-we-do/survey/survey-display-451.cfm. Accessed November 5, 2015.

4. Scheiwe A, Hardy R and RG Watt Four-year follow-up of a randomized controlled trial of a social support intervention on infant feeding practices. Matern Child Nutr. 2010; 6: 328-337.

5. WHO/UNICEF Complementary feeding of young children in developing countries: a review of current scientific knowledge. Geneva: World Health Organization, WHO/NUT/98.1, 1998.

6. Whyte SR and PW Kariuki Malnutrition and gender relations in Western Kenya. Health Transit Rev. 1991; 1: 171-187.

7. Ssemukasa EL and J Kearney "Complementary feeding practices in Wakiso district of Uganda". African Journal of Food, Agriculture, Nutrition and Development (2014);14.4: 9085-9103.

8. Owino VO, Amadi B, Sinkala M, Filteau S and A Tomkins "Complementary feeding practices and nutrient intake from habitual complementary foods of infants and children aged 6-18 months old in Lusaka, Zambia." African Journal of Food, Agriculture, Nutrition and Development 8.1 (2008): 28-47.

9. Kerr RB, Dakishoni L, Shumba L, Mschai $\mathbf{R}$ and $\mathbf{M}$ Chirwo We grandmothers know plenty: Breastfeeding, complementary feeding and the multifaceted role of grandmothers in Malawi, Soc Sci Med. 2008; 66: 10951105 .

10. Mukuria AG Exclusive breastfeeding and the role of social support and social networks in a low income urban community in Nairobi, Kenya. DrPH dissertation. Baltimore, Maryland: Johns Hopkins University Bloomberg School of Public Health; 1999.

11. Aubel J, Toure I and M Diagne Senegalese grandmothers promote improved maternal and child nutrition practices: the guardians of tradition are not averse to change. Soc Sci Med. 2004; 59: 845-859. 


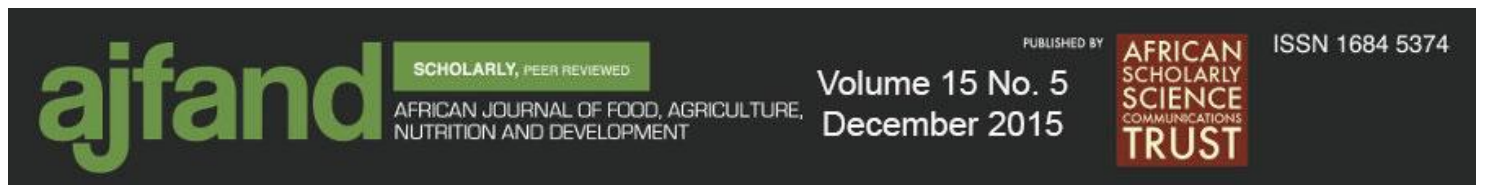

12. Aubel J The role and influence of grandmothers on child nutrition: culturally designated advisors and caregivers. Matern Child Nutr. 8, 19-35.

13. Susin LR and ER Giugliani Inclusion of fathers in an intervention to promote breastfeeding: impact on breastfeeding rates. J Hum Lact. 2008; 24: 386-392.

14. Pisacane A, Continisio GI, Aldinucci M, D'Amora S and P Continisio A controlled trial of the father's role in breastfeeding promotion. Pediatrics. 2005; 116: e494-e498.

15. Kuyper E and K Dewey Literature Review: Fathers support infant and young child feeding: Their contributions to better outcomes. Washington, DC: FHI 360,2012

http://www.aliveandthrive.org/sites/default/files/Lit\%20review_Final_2.pdf. Accessed $11^{\text {th }}$ Aug, 2014.

16. Sloand E, Astone NM and B Gebrian The Impact of Fathers' Clubs on Child Health in Rural Haiti. Am J Public Health. 2010; 100: 201-204.

17. Israel-Ballard $\mathbf{K}$, Waithaka $\mathbf{M}$ and $\mathbf{T}$ Greiner Infant feeding counselling of HIV-infected women in two areas in Kenya in 2008. Int J STD AIDS. 2014 Epub Mar 4.

18. Thuita FM Infant and young child feeding practices in Kenya: a review of the literature. Nairobi, Kenya: PATH, 2008.

19. Nduati R, Arum $\mathbf{S}$ and $\mathbf{E}$ Kageha Beliefs and attitudes around infant and young child feeding in Kenya: findings from a rapid qualitative assessment. Nairobi, Kenya: PATH, 2008.

20. United Nations Children's Fund (UNICEF) and Government of Kenya Qualitative assessment of infant feeding practices in 10 UNICEF focus districts. Nairobi, Kenya: UNICEF, 2007.

21. Republic of Kenya National Strategy on Infant and Young Child Feeding 2007 to 2010. World Health Organization and UNICEF, 2007.

22. Simons-Morton B Health behavior in ecological context. Health Educ Behav. 2013; 40: 6-10.

23. USAID's Infant and Young Child Nutrition Project (IYCN) Engaging grandmothers and men in infant and young child feeding and maternal nutrition: Report of a formative assessment in Eastern and Western Kenya. Washington, DC: IYCN; 2011 http://iycn.wpengine.netdna-cdn.com/files/IYCN_KenyaEngaging-Grandmothers-and-Men-Formative-Assessment_0511.pdf. Accessed August 11, 2014. 
24. Martin S, Muhomah T, Thuita F, Bingham A and A Mukuria What motivates maternal and child nutrition peer educators? Experiences of fathers and grandmothers in western Kenya. Soc Sci \& Med. 2015; 143: 45-53.

25. USAID's Infant and Young Child Nutrition Project (IYCN) Engaging grandmothers to improve nutrition: A training manual for dialog group mentors. Washington, DC: PATH, 2011 http://iycn.wpengine.netdnacdn.com/files/FINAL-IYCN-GM-Training-071411.pdf. Accessed August 11, 2014.

26. USAID's Infant and Young Child Nutrition Project (IYCN) Infant and young child feeding and gender: A training manual for male group leaders Washington, DC: PATH, 2011 http://iycn.wpengine.netdna27cdn.com/files/IYCN_Mens-Groups-IYCF-Training-Manual_0311.pdf. Accessed August 11, 2014.

27. USAID's Infant and Young Child Nutrition Project (IYCN) Infant and young child feeding and gender: A participant manual for male group leaders Washington, DC: PATH, 2011 http://iycn.wpengine.netdnacdn.com/files/IYCN_Mens-Groups-IYCF-Participant-Manual_0311.pdf. Accessed August 11, 2014.

28. World Health Organization, UNICEF Infant young child feeding counselling: An integrated course. 2006 http://www.who.int/nutrition/publications/infantfeeding/9789241594745/en. Accessed August 11, 2014.

29. Britton C, McCormick FM, Renfrew MJ, Wade A and SE King Support for breastfeeding mothers. Cochrane Database Syst Rev. 2007; 1.

30. Chapman DJ, Morel K, Anderson AK, Damio G and R Pérez-Escamilla Review: Breastfeeding peer counseling: from efficacy through scale-up. J Hum Lact. 2010; 26: 314-326.

31. Aubel $\mathbf{J}$ The roles and influence of grandmothers and men. Evidence supporting a family focussed approach to optimal infant and young child nutrition. A review of literature. Washington, DC: USAID's Infant and Young Child Nutrition Project (IYCN), 2012: 30-31.

32. USAID's Infant and Young Child Nutrition Project (IYCN) The roles and influence of grandmothers and men: Evidence supporting a family-focused approach to optimal infant and young child nutrition. Washington, DC: PATH, 2011 http://iycn.wpengine.netdna-cdn.com/files/IYCN-GM-and-Men-LitReview-060311.pdf. Accessed August 11, 2014.

33. Ovretveit J Evaluating Health Interventions. Buckingham: Open University Press, 1998. 


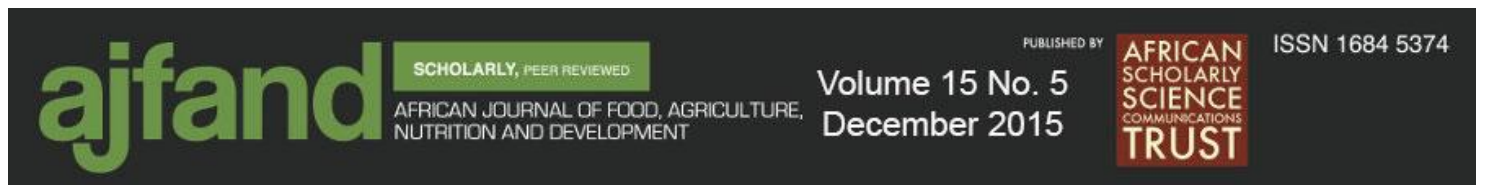

34. Fabrizio CS, van Liere $\mathbf{M}$ and $\mathbf{G}$ Pelto Identifying determinants of effective complementary feeding behaviour change interventions in developing countries. Matern Child Nutr. 2014; May 5 [Epub ahead of print] http://www.ncbi.nlm.nih.gov/pubmed/24798264. Accessed 10th Sept 2014.

35. Bentley ME, Johnson SL, Wasser H, Creed-Kanashiro H, Shroff M, Fernandez Rao $\mathbf{S}$ and $M$ Cunningham Formative research methods for designing culturally appropriate, integrated child nutrition and development interventions: an overview. Ann N Y Acad Sci. 2014; 1308: 54-67. http://onlinelibrary.wiley.com/doi/10.1111/nyas.12290/abstract. Accessed August 11, 2014.

36. Bingham A, Kidwell Drake J, Goodyear L, Gopinath CY, Kaufman A and S Bhattarai The Role of Interpersonal Communication in Preventing Unsafe Abortion in Communities: The Dialogues for Life Project in Nepal. J Health Commun. 2011; 16: 245-263.

37. Figueroa ME, Kincaid DL, Manju R and G Lewis Communication for social change: An integrated model for measuring the process and its outcomes. New York: The Rockefeller Foundation, 2002.

38. Duggan A Understanding interpersonal communication processes across health contexts: Advances in the last decade and challenges for the next decade. $J$ Health Commun. 2006; 11: 93-108.

39. Valente TW and $\mathbf{R}$ Fosados Diffusion of innovations and network segmentation: the part played by people in promoting health. Sex Transm Dis. 2006; 33: S23-31.

40. Pelto $\mathbf{G}$ and $\mathbf{M}$ Armar-Klemesu. Identifying interventions to help rural Kenyan mothers cope with food insecurity: results of a focused ethnographic study. Mat \& child nut. in press.

41. Dykes F Government funded breastfeeding peer support projects: implications for practice. Matern Child Nutr. 2005; 1: 21-31. 